\title{
LA-8458-MS
}

Informal Report

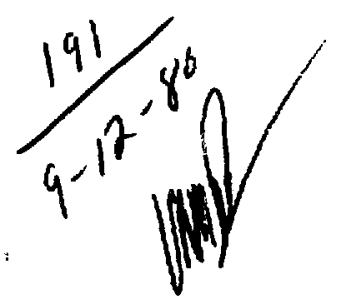

\section{One-Dimensional Computer Simulations of Exploding Pusher Targets for the Los Alamos Helios Laser System}

\section{MASTER}

6

5

\%

$\frac{2}{9}$

市

$\stackrel{\text {. }}{\text { I }}$

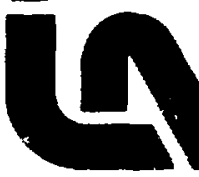




\title{
One-Dimensional Computer Simulations of Exploding Pusher Targets for the Los Alamos Helios Laser System
}

\author{
William P. Gula
}

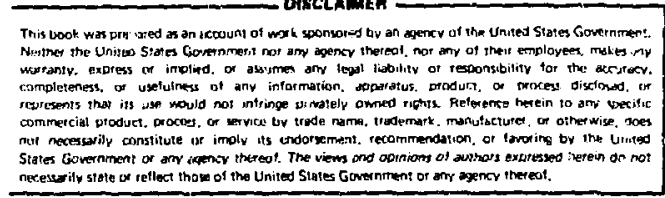




\title{
ONE-DIMENSIONAL COMPUTER SIMULATIONS OF EXPLODING PUSHER \\ TARGETS FOR THE LOS ALAMOS HELIOS LASER SYSTEM
}

\author{
by \\ Hilliam P. Gula \\ ร \\ ABSTRACT \\ This report summarizes the results of a series \\ of one-dimensional computer simulations done at Los \\ Alamos for 10.6- $\mu \mathrm{m}$ laser pulses with peak powers in \\ the 5-20 TW range on exploding pusher tarcets. \\ These calculations were done in order to predict \\ and understand the first experimental results on \\ the Helios $\mathrm{CO}_{2}$ eight-beam laser systen.
}

\section{INTRODUCTION}

Now that the Helios $\mathrm{CO}_{2}$ eight-beam laser system is being used for target experiments, we need to have some calculations of the perf: mance expected from various targets. Accordingly, we have performed numerous computer simulations. Much work has been published on the behaviour of exploding pusher targets, 1-5 but this is the first to deal with the $10.6-\mu \mathrm{m} \mathrm{CO}_{2}$ wavelength at powers of 5-20 TW. This report is in the same spirit as Cranfill's work ${ }^{4}$ and may be considered as an extension to $\mathrm{j} \%$.

The two main purposes for this study are first, to aid in the design and interpretation of experiments on the Los Alamos Helios eight-beam $\mathrm{CO}_{2} \mathrm{laser}$ system and second, to calibrate the computer code with the experimental results.

Single shell DT filled giass microballoons were chosen for this study because 1) they are relatively simple and have a relatively small number of parameters that can be varied, 2) the targets are easy to diagnose 
experimentally and, 3) we think we understand the physics of these exploding pusher type targets.

The simulations were one-dimensional calculations in which the glass shells were perfect spheres with an initial density of $2.4 \mathrm{gm} / \mathrm{cm}^{3}$ filled with an equimolar mixture of deuterium and tritium.

Most of the calculations were done using a pulse shape of the form

$$
P=P_{n} \exp (-\alpha t)[1-\exp (-\beta t)]^{2}
$$

where $P_{n}, \alpha$, and $B$ are adjustable parameters. This pulse shape was chosen because it provides a good fit to the pulse shape of the Los Alamos two-beam $\mathrm{CO}_{2}$ laser system. We feel that this function should also provide a good fit to the pulse shape of the Helios laser system, especially since the Helios amplifiers are basically improved versions of the two beam systems's amplifiers. In actual practice the parameters $P_{p}$, the peak laser power, $\tau_{R}$, the laser risetime $(0-100 \%)$, and the energy width $\Delta T\left(=E / P_{p}\right.$ where $E$ is the total energy) are given and the computer then calculates $\alpha, \beta$ and $P_{n}$.

In this study we varied the radius of the glass shell, the thickness of the glass shell, the DT fill pressure, the peak laser power, the laser rise time, and the percentage of neon mixed in with the fuel. Some work was also done varying the energy width $\Delta \mathrm{T}$.

The general range of the parameters considered was

$\begin{array}{ll}\text { Peak power } & 5-20 \mathrm{TW} \\ \text { Rise time } & 100-300 \mathrm{ps} \\ \text { Shell inner radius } & 50-400 \mu \mathrm{m} \\ \text { Shell thickness } & 0.75-1.75 \mu \mathrm{m} \\ \text { Initial fill pressure } & 1-30 \mathrm{~atm} .\end{array}$

II. NEUTRON CALCULATIONS

Figure 1 shows both pulse shapes that were used, the $\mathrm{CO}_{2}$ pulse given by Eq. (1) and the triangular equivalent. In this figure both pulses have the same 0-100\% risetime (200-ps), the same peak power (5 Tw) and the same energy (5 kJ). Figure 2 shows the results of varying the pulse shape and also the shell radius 


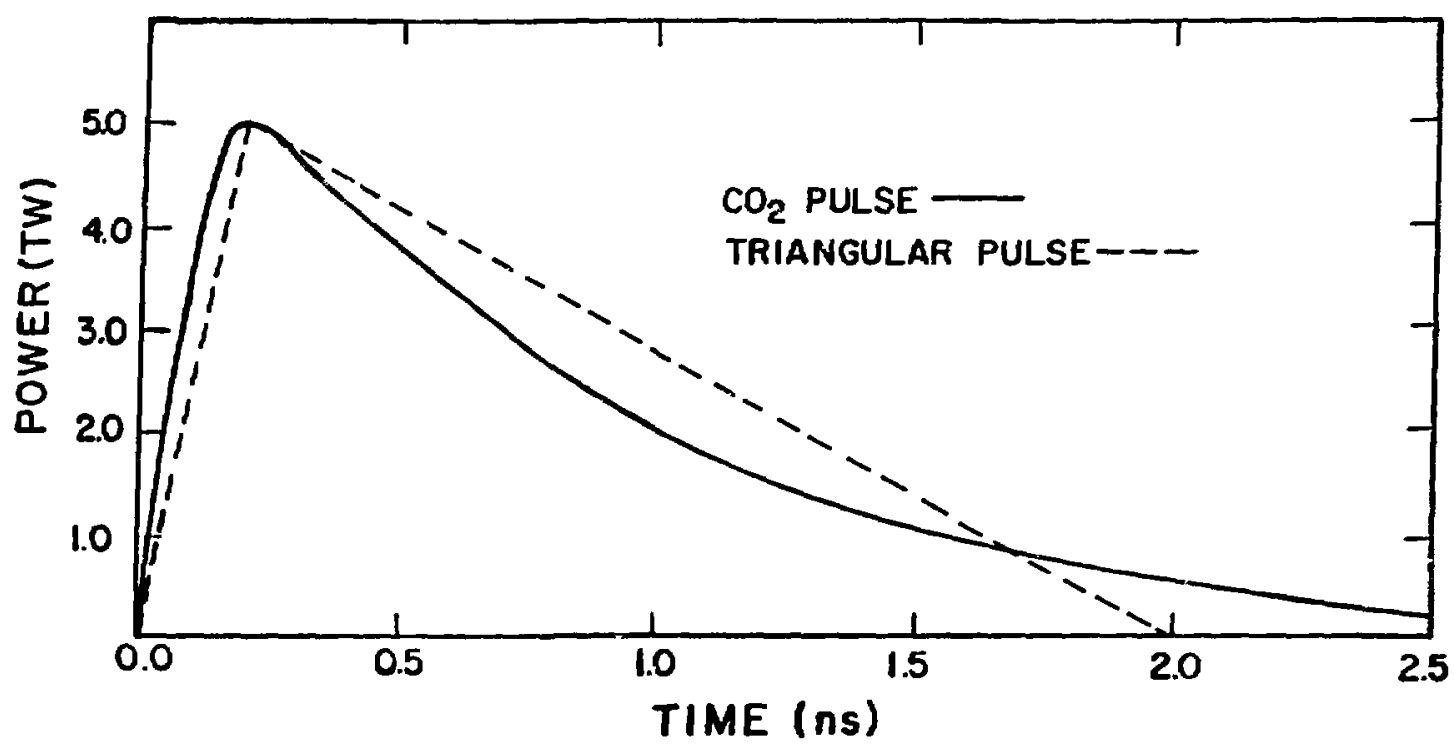

Eif. 1. The power in the two different laser pulse shapes as a function of time. Both pulses have the same 0-100\% risetime (200-ps), the same peak power ( $5 \mathrm{TH})$, and the same total energy $(5 \mathrm{~kJ})$.

for a given power. This figure shows that for a given peak power the $\mathrm{CO}_{2} \mathrm{pulse}$ always gives better results than the triangular pulse for the cases studied. This is because the $\mathrm{CO}_{2}$ pulse delivers more energy earlier for the same 0-100\% risetime. Another way of stating this is that, although the 0-100\% risetime of the two pulses is identical, the $0-80 \%$ risetime of the $\mathrm{CO}_{2}$ pulse is shorter ( $2 / 3$ ) than the triangular $0-80 \%$ risetime. For the cases shown in Fig. 2, the total energies were the same for the $\mathrm{CO}_{2}$ and triangular pulses for the 5 TW $(5 \mathrm{~kJ}), 10 \mathrm{TW}(10 \mathrm{~kJ})$, and $15 \mathrm{TW}(7.5 \mathrm{~kJ})$ runs. For the $20 \mathrm{TW}$ cases the $\mathrm{CO}_{2}$ pulse energy was $7.4 \mathrm{~kJ}$ and the triangular pulse energy was $5 \mathrm{~kJ}$.

Figure 3 shows the predicted yield as a function of target radius for different pulse widths. All the calculations shown on Fig. 3 are for the $\mathrm{CO}_{2}$ laser pulse shape with a 200-ps risetime. The calculations are grouped into four pairs. Each pair has the same peak power, but the upper member of each pair has twice the energy. In general Fig. 3 shows that the peak power is more important than the total energy or that it is important to get the energy in early. Although in all cases an increase in total energy improves the yield. the difference is not substantial except for the larger targets which, because of their longer run-in time, are able to make use of the energy that comes laters 


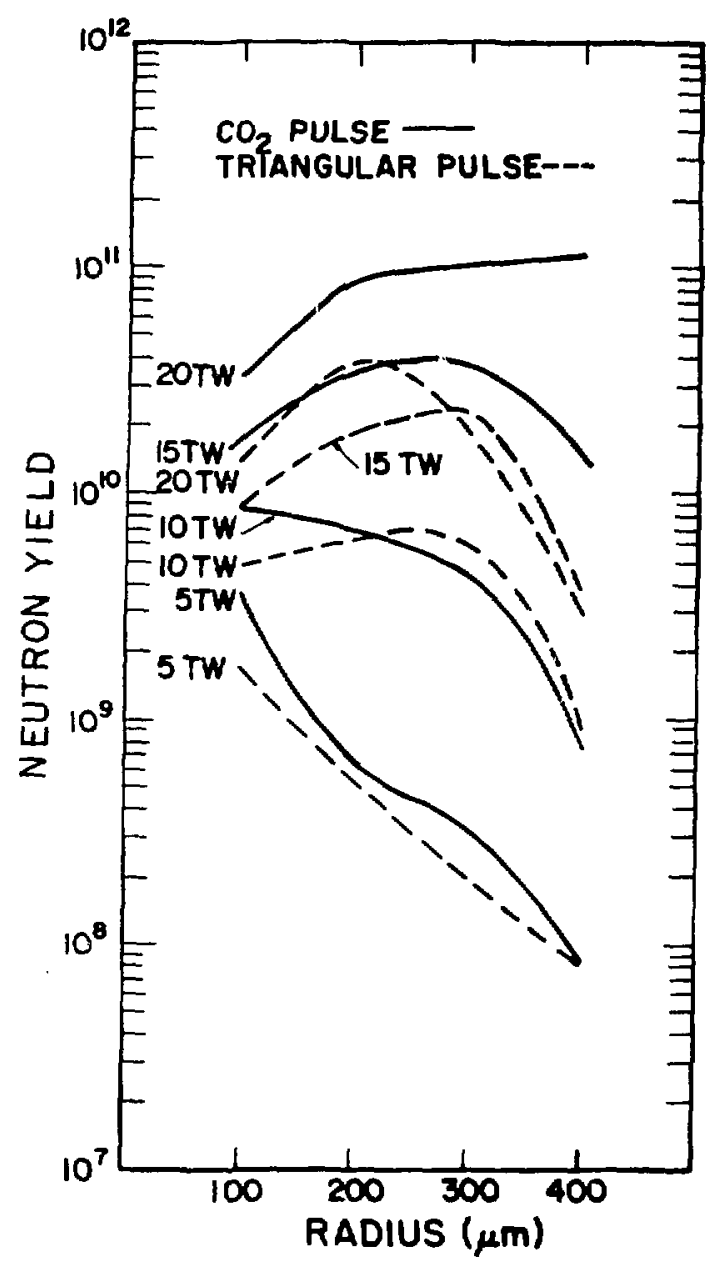

Fig. 2. Neutron vield vs peak laser power for selected targets of different shell inner radius with different laser pulse shapes. All targets had a shell thickness of $1 \mathrm{um}$ and a DT fill pressure of 10 atm. All the laser pulses had a risetime of $200 \mathrm{ps}$ and an energy width of 1 ns.

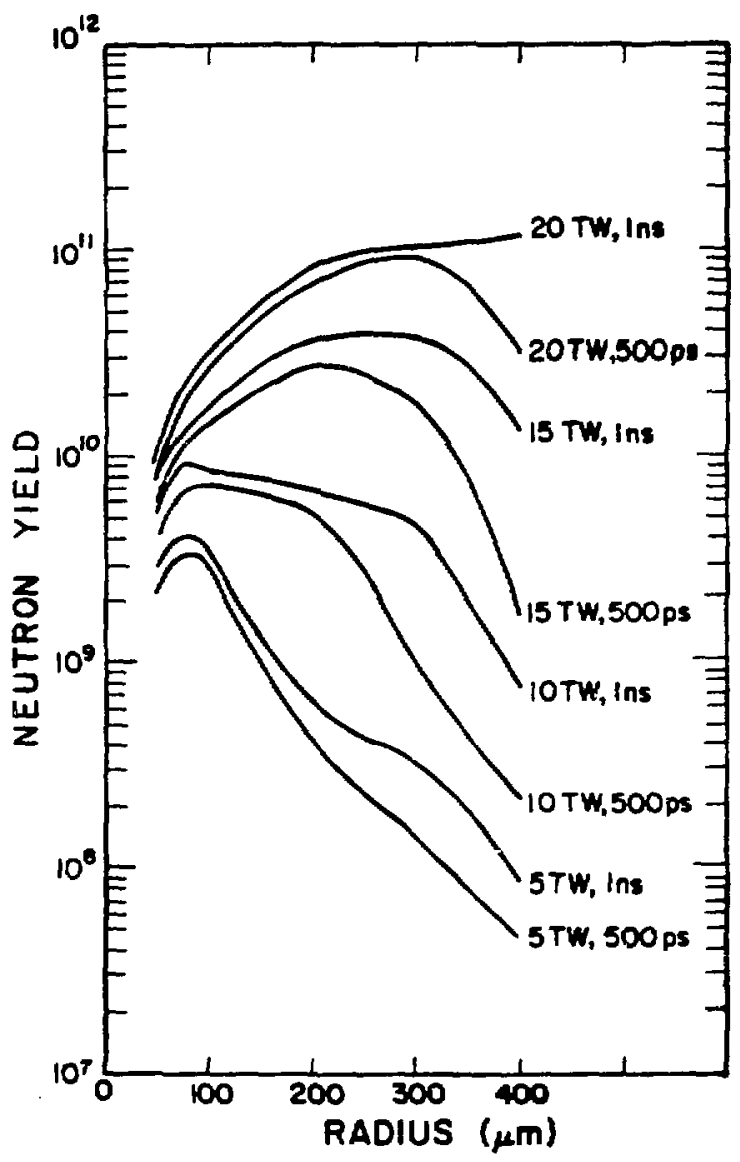

Fig. 3. Neutron yield vs target radius for different laser pulse widths. All the laser pulses had the $\mathrm{CO}_{2}$ laser pulse shape with a 200-ps risetime. All the targets had a shell thickness of $1 \mu \mathrm{m}$ and a DT fill pressure of 10 atm.

in the pulse. Even in these cases we should note that except for the $20 \mathrm{TW}$ curves the advantage of the higher energy pulse becomes substantial at off optimal radii. This seems to be because the larger radius is needed to take advantage of the energy delivered late in the pulse means that there is more ruel mass to heat to fusion temperature and more glass mass to heat and explode 
(all these calculations were done for a constant fill pressure of 10-atm and a constant glass shell thickness of $1-\mu \mathrm{m})$. The extra energy at the lower powers is not enough to overcome the difficulty in heating the extra fuel. A more dramatic illustration of the importance of getting the energy in early is the comparison of curves $10 \mathrm{TW}, 500-\mathrm{ps}$ and $5 \mathrm{TW}, 1 \mathrm{~ns}$, which each have a total of $5 \mathrm{~kJ}$, and curves $20 \mathrm{TW}, 500-\mathrm{ps}$ and $10 \mathrm{TW}, 1 \mathrm{~ns}$, which each have a total energy of $10 \mathrm{~kJ}$. The upper member of each pair has twice the peak power and from 2 to 40 times the neutron yield for the same target. As with all rules, however, there are some exceptions. Figure 4 shows the neutron yield for various targets as a function of pulse risetime. In general, the faster the risetime the better. However, for large targets and higher powers the shorter risetime evidently results in more target preheat before the shell explosion can compress the fuel effectively.

Figure 5 shows the neutron yield as a function of laser peak power for a given target and pulse width. The steepness of these curves emphasizes the importance of the dependence on peak power for the exploding pusher targets in this regime. The exceptions are the curves for the $\leq 100-\mu m$ radius targets. Their slopes are much lower than the others. This lower slcpe is consistent with the curve shown in Fig. II. 5 of Ref. 4. These smaller targets are

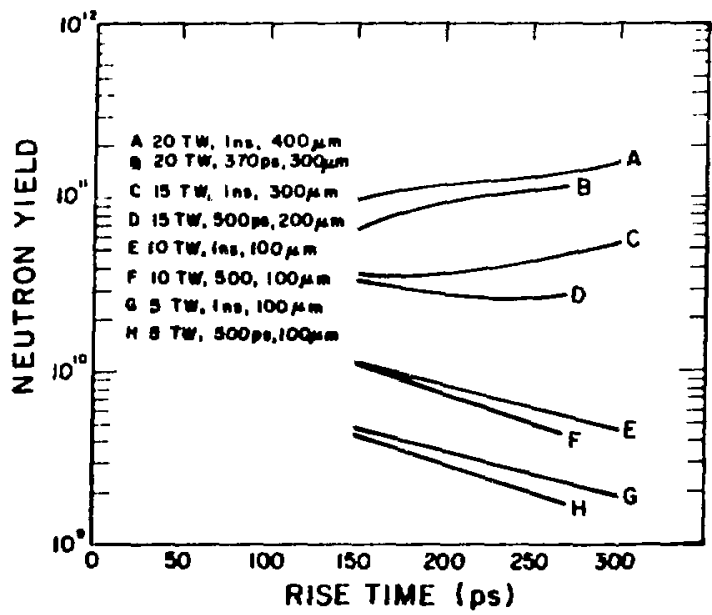

Fig. 4. Neutron yield vs laser pulse risetime for selected targets and laser peak powers. All targets had a shell thickness of $I \mu \mathrm{m}$ and a DT fill pressure of $10 \mathrm{~atm}$. All laser pulses were the $\mathrm{CO}_{2}$ laser pulse shape. saturated at these powers. Any extra power (energy) that is absorbed efficiently converted into fuel heating.

Another variable considered was the initial fill pressure. Figure 6 shows how the neutron yield varied as a function of fill pressure for specific targets and laser pulses. The most interesting feature about this figure is that the curves turn down as the fill pressure goes to 1-atm. This is interestine because, although we know that the yield must go down 
at some point as we reduce the fill pressure (and therefore the fuel mass) this had not shown up in earlier target simulations.

Futhermore, although the yields are lower at 1-atm they are still substantial $\left(\sim 10^{9}\right)$ and thus we can test these curves experimentally. Figure 7 shows the neutron yield as a function of shell thickness for selected target radii and laser pulses. The 5 and 10 TW curves show a weak dependence on shell thickness over the range considered. The $10 \mathrm{TW}$ curve shows a broad maximum.

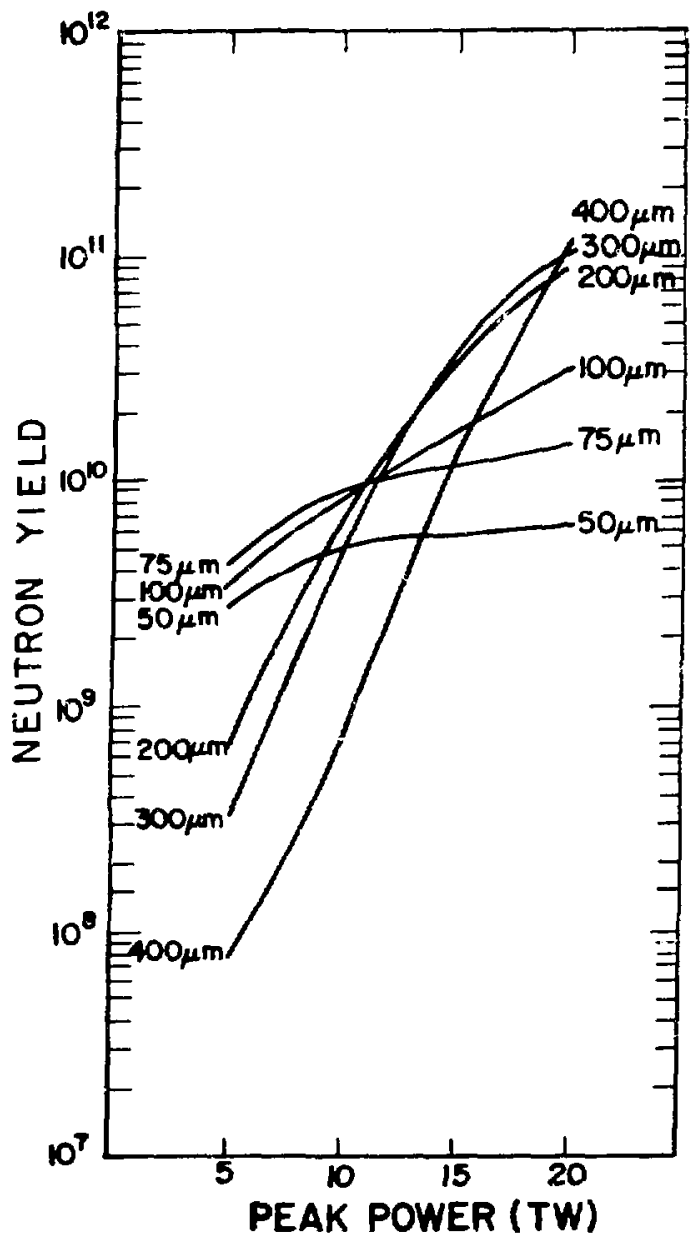

Fig. 5. Neutron yield vs peak power for selected target radii. All the laser pulses had the $\mathrm{CO}_{2}$ laser pulse shape, a 200-ps risetime, and a 1-ns pulse width. All the targets had a shell thickness of $1 \mu \mathrm{m}$ and a DT fill pressure of 10 atm.

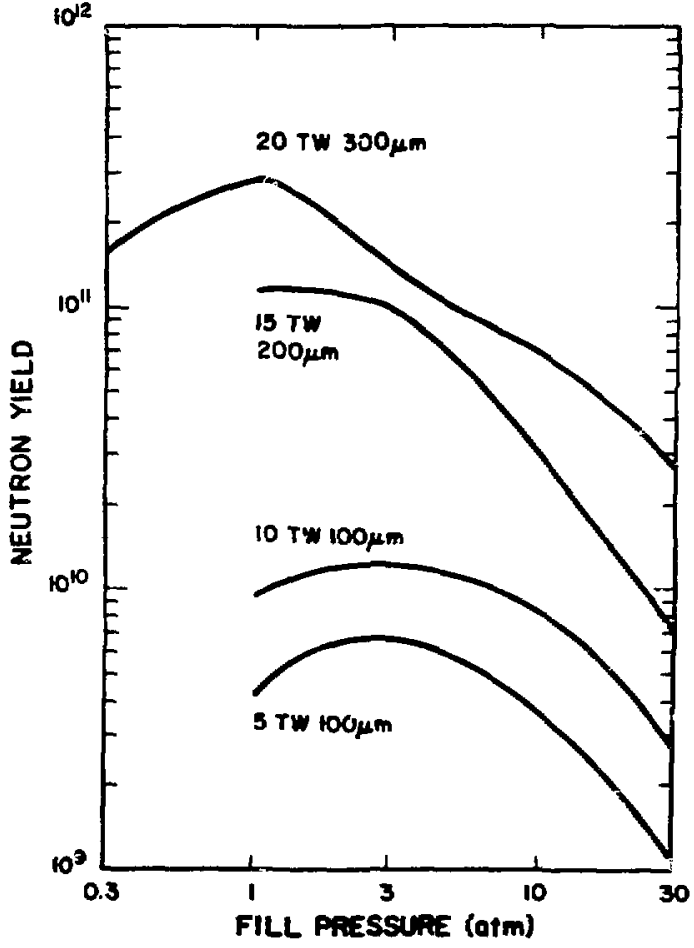

Fig. 6. Neutron yieid vs DT fill pressure for selected targets and laser pulse peak powers. The laser pulses had the $\mathrm{CO}_{2}$ pulse shape with a 200-ps risetime and a 1-ns pulse width. The targets had a shell thickness of $1 \mu \mathrm{m}$. 


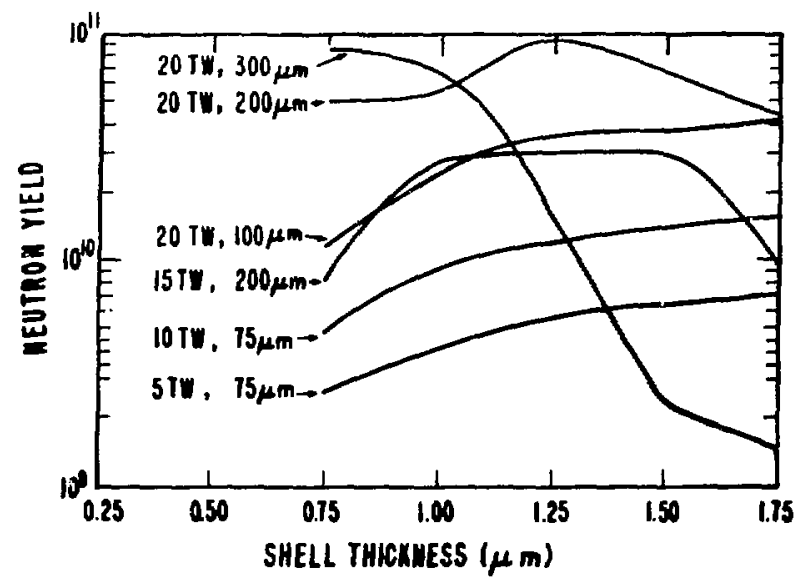

Fig. 7. Neutron yield as a function of shell thickness for selected target radii and laser peak powers. The plaser pulses are 200-ps risetime 1 ns wide $\mathrm{CO}_{2}$ pulses. The targets had a DT fill pressure of $10 \mathrm{~atm}$.

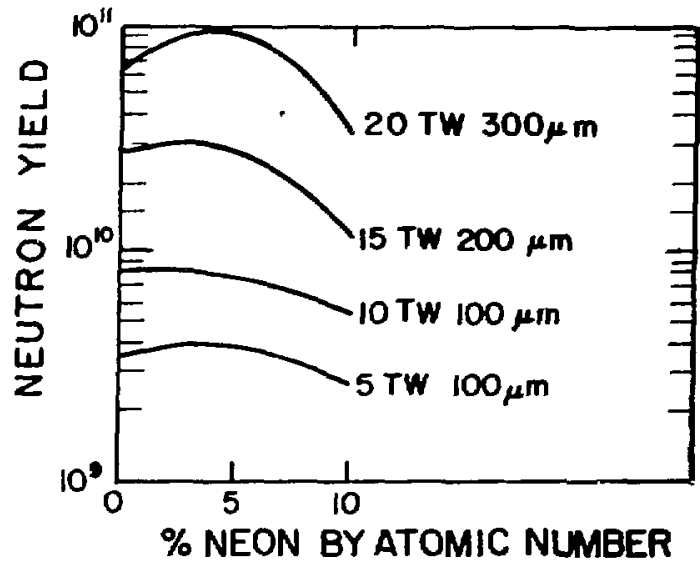

Fig. 8. Neutron yield as a function of the percentage of neon by atomic number mixed in the fuel for four different targets and laser peak powers. The laser pulses are 200-ps risetime 1 ns wide $\mathrm{CO}_{2}$ pulses. The targets have a shell thickness of $1 \mu \mathrm{m}$ and the same number of ions as that of a 10-atm DT fill pressure.

thickness over the range considered. The $10 \mathrm{TW}$ curve shows a broad maximum. Examination of the $20 \mathrm{TW}$ curves shows that the position of the maximum yield as a function of shell thickness also depends fairly strongly on the radius. In fact it would seem that the most important considerations here are the peak power (actually total usable energy absorbed is even better) and the mass of the shell. Looking at the $20 \mathrm{TW}, 200-\mu \mathrm{m}$ curve we see a maximum at a shell thickness of 1.25- $\mu \mathrm{m}$. The corresponding maxima for the $200 \mathrm{TV}: 300-\mu \mathrm{m}$ and $250 \mathrm{TW} 100-\mu \mathrm{m}$ curves should be at $0.6-\mu m$ and $5.0-\mu m$ resf zctively if we accept the idea that the shell mass is the important variable. The actual curves do not contradict this assumption.

Figure 8 shows the neutron yield as a function of the percentage of neon in the fuel for four different targets and pulses. This is of interest because neon is sometimes put into the DT fuel for diagnostic reasons. At lower $\mathrm{CO}_{2}$ laser powers (:5 TW) computer simulations have predicted that adding neon would increase the yield while the experiments have shown a degradation of yield with 
either makes little difference or will degrade the yield. Again even the lower yields are above $10^{9}$ neutrons and so the curves should be subject to experimental verification.

\section{COMPARISON WITH EXPERIMENTAL RESULTS}

In the initial exploding pusher experiments of Helios, there were six shots for wich pulse shapes were available. These proved to be approximately trapezoidal. Table I gives the target and laser parameters and both the experimental and calculated neutron yield. In gerieral, the calculated values agree remarkably well with the measured values. On average the calculated yields are a factor of 1.5 lower than the measured yields. The obvious exception to this statement is shot number 89010405 . Table 1 also includes a comparison of the simulations with the simple model of Giovanielli and Cranfill. 6 The agreement is impressive.

\section{CONCLUSIONS}

The first and most important result to note is that simulations predict that with any reasonable exploding pushing target the Helios laser system should produce at least $10^{8}$ neutrons. A "good" target should produce $2 \times 10^{9}$ at $10 \mathrm{TW}$.

The second point is that even the not so good targets are predicted to produce measureable amounts of neutrons. Therefore the theoretical curves of variation of yield with power, risetime, fill pressure, radius, and neon percentage can be subject to experimental verification.

\section{REFERENCES}

1. G. S. Fraley and R. J. Mason, Phys. Rev. Lett. 35, 520 (1975).

2. G. Charatis, J. Downward, R. Goforth, B. Guscott, T. Henderson, J. Hildum, F. Johnson, K. Moncur, T. Leonard, F. Mayer, S. Segall, L. Liebert, B. Solomon, and C. Thomas, Plasma Physics and Controlled Nuclear Fusion Research (Proc. Int. Conf., Tokyo, 1974) IAEA, Vienna, Vol. II, p. 317.

3. J. H. Nuckolls, Lawrence Livermore Laboratory Report No. UCRL-79834 (1977).

4. C. W. Cranfill, Los Alamos Scientific Laboratory Report No. LA-6827-MS (1977). 
5. M. D. Rosen and J. H. Nuckolls, Phys. of Fluids, 22, 1393 (1979).

6. D. V. Giovanielli and C. W. Cranfill, Los Alamos Scientific Laboratory Report No. LA-7218-MS (1978). 
TARLF I

NEUTRON YIFLD AS A FUNCTION OF TARGET AND LASER PARAMETERS

\begin{tabular}{|c|c|c|c|c|c|c|c|c|c|c|c|}
\hline \multirow{2}{*}{$\begin{array}{c}\text { Shot } \\
\text { Number }\end{array}$} & \multicolumn{3}{|c|}{$\begin{array}{c}\text { Target } \\
\text { Parameters }\end{array}$} & \multicolumn{4}{|c|}{$\begin{array}{l}\text { Equivalent Trapezuidal } \\
\text { Shaped Laser Pulse }\end{array}$} & \multirow{2}{*}{$\begin{array}{l}\text { Measured } \\
\text { Neutron } \\
\text { Yield } Y_{\mathrm{m}} \\
\left(\times 10^{8}\right)^{2}\end{array}$} & \multirow{2}{*}{$\begin{array}{l}\text { Calculated } \\
\text { Neutron } \\
\text { Yield }{ }^{Y} \mathrm{c} \\
\left(x \quad 10^{8}\right)^{-}\end{array}$} & \multirow{2}{*}{$\frac{Y_{r_{r}}}{Y_{c}}$} & \multirow{2}{*}{$\frac{Y_{c}}{Y_{s}}$} \\
\hline & $r(\mu m)$ & $\Delta r(\mu m)$ & $\begin{array}{c}\text { DT fill } \\
\text { Pressure(atm) }\end{array}$ & ${ }^{\top}$ rise $(p s)$ & $\tau^{\tau}$ flat $(p s)$ & $T_{\text {fall }}$ (ps) & $\begin{array}{c}\text { Peak } \\
\text { Power(TW) }\end{array}$ & & & & \\
\hline 88110705 & 172.5 & 1.57 & 9 & 471 & 68 & 636 & 2.30 & 0.41 & 0.59 & 0.69 & 1.44 \\
\hline 88110706 & 217 & 0.98 & 10 & 320 & 71 & 989 & 3.40 & 2.3 & 1.1 & 2.0 & 0.20 \\
\hline 88110708 & 189 & 0.88 & 8 & $36 n$ & 110 & 763 & 3.47 & 3.5 & 1.9 & 1.8 & 0.45 \\
\hline 88110709 & 198 & 1.18 & 10 & 375 & 198 & 729 & 2.45 & 1.1 & 0.70 & 1.6 & 0.56 \\
\hline 89010405 & 98.5 & 1.11 & 9 & 221 & 97 & 1191 & 6.39 & 0.40 & 24.7 & 0.02 & 2.38 \\
\hline 89032004 & 149.5 & 0.97 & 28 & 257 & 222 & 1118 & 4.21 & 2.1 & 1.7 & 1.2 & 0.31 \\
\hline
\end{tabular}

" ${ }_{s}$ Neutron yield calculated by the model in Ref. 6. 